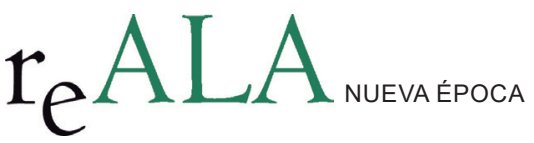

REALA, Nueva Época - N. ${ }^{\circ}$, enero-junio 2016 - ISSN: 1989-8975

DOI: http://dx.doi.org/10.24965/reala.voi5.10347

Rafael García Matíes

Fundación Musol de Cooperación Internacional para el Desarrollo

musol@musol.org

\title{
Las entidades locales y los objetivos de desarrollo sostenible. Algunas notas sobre la naturaleza jurídica de la Agenda 2030
}

\section{Resumen}

La Asamblea General de Naciones Unidas aprobó, en la reunión plenaria de los días 25 al 27 de septiembre de 2015, una declaración denominada: "Transformar nuestro mundo: La Agenda 2030 para el Desarrollo Sostenible». España y 192 países más han suscrito esa Agenda, que ha entrado en vigor el 1 de enero de 2016. La Agenda se configura como un plan de acción, a favor de las personas, el planeta y la prosperidad y se refiere a 17 objetivos y 169 metas a alcanzar en todo el planeta, estableciendo que todos los actores públicos y privados, hemos de trabajar para su logro. Se analiza la naturaleza jurídica de la declaración y el papel de los municipios en el ámbito internacional, singularmente en la implementación de la agenda.

Palabras clave

ONU, Agenda 2030, ODS, desarrollo sostenible, objetivos, metas, competencias, naturaleza jurídica, soft law, Tratados internacionales, acuerdos internacionales no normativos, alcance de las declaraciones de los organismos internacionales.

\section{Local authorities and sustainable development aims. Some notes on the legal nature of the 2030 Agenda}

\section{Abstract}

The United Nations General Assembly adopted, at the plenary meeting from 25 to 27 September 2015, a declaration entitled «Transforming Our World: The 2030 Agenda for Sustainable Development». Spain, along with more than 192 countries, has signed the Agenda which came into effect on 1 January 2016. The Agenda is configured as an action plan for people, the planet, and prosperity and relates to 17 objectives and 169 goals to achieve across the globe, establishing that all public and private actors have to work for their attainment. The UN analyzed the legal nature of the declaration and the role of municipalities on the international level, especially in the implementation of the agenda.

\section{Keywords}

United Nations, 2030 agenda, Sustainable Development Goals (SDG), sustainable development, SDGs targets, Local authorities competences, legal status, soft law, international treaties, not binding international agreements, scope of the international declarations. 


\section{INTRODUCCIÓN}

Tradicionalmente los municipios y demás entes locales se han visto excluidos por la legislación, de participar y actuar en cuanto ocurría fuera de su término municipal. Sus competencias, dice el artículo 12 de la Ley 7/1985 de 2 de abril, Reguladora de las Bases del Régimen Local (en adelante LRBRL), se extienden al término municipal.

Las actuaciones de los municipios y del resto de los entes locales, en el campo internacional, se han visto como algo que no concernía más que al Estado. Las leyes 23/1998 de 7 de julio de Cooperación Internacional para el Desarrollo y 2/2014, de 25 de marzo, de la Acción y del Servicio Exterior del Estado, suponen un punto de inflexión muy importante, al reconocer a los municipios y demás entes locales protagonismo en la acción exterior del Estado, aun con bastantes limitaciones.

En muchas ocasiones, cuando los representantes municipales han tratado de relacionarse con el exterior, se les ha negado esta facultad. Recientemente por algunos, se ha discutido la competencia de los municipios, para llevar a cabo actuaciones de Cooperación Internacional para el Desarrollo, alegando que la Competencia en Cooperación Internacional para el Desarrollo no era una competencia propia municipal después de la modificación de la $\mathrm{LRBRL}^{1}$, sin tener en cuenta, que esa actividad se venía ejerciendo pacíficamente, al amparo del artículo 20 de la Ley 23/1997, de Cooperación que sigue vigente ${ }^{2}$. La reciente sentencia del Tribunal Constitucional de 3 de marzo de 2016, ha venido a confirmar que las competencias propias atribuidas por la legislación sectorial no han sido derogadas por el solo hecho de no figurar ahora en el listado del artículo 25 de la LRBRL.

Todavía hoy, más allá de los Hermanamientos y de la Cooperación Internacional para el Desarrollo, la mayor parte de los municipios, no han interiorizado que tienen un rol a desempeñar en el exterior y ello pese a que la Ley 2/2014, de 25 de marzo, de la Acción y del Servicio Exterior del Estado, se refiere en alguno de sus preceptos, expresamente a la Administración Local, reconociendo a todas las administraciones públicas, incluida la local, como sujetos de la Acción Exterior, atribuyéndoles la facultad de efectuar visitas e intercambios, facultándoles para celebrar acuerdos no normativos con los órganos análogos de otros sujetos de derecho internacional, no vinculantes jurídicamente, para quienes los suscriben, sobre materias de su competencia y facultándoles para efectuar aportaciones a la planificación en los artículo 1, 5, 11 y 34 y siguientes. Recordemos que conforme el artículo 2 de la Ley 25/2014, de 27 de noviembre de Tratados y otros Acuerdos Internacionales. Los acuerdos internacionales no normativos, son acuerdos que contienen declaraciones de intenciones o establecen compromisos de actuación de contenido político, técnico o logístico y no constituye fuente de obligaciones internacionales, ni se rige por el Derecho Internacional.

El artículo 52.1 de la Ley 25/2014, de 27 de noviembre, de Tratados y otros Acuerdos Internacionales, faculta también a las entidades locales para suscribir acuerdos internacionales administrativos sobre materias de su competencia, al establecer que:

«Las Comunidades Autónomas podrán celebrar acuerdos internacionales administrativos en ejecución y concreción de un tratado internacional cuando tengan por ámbito materias propias de su competencia y con sujeción a lo que disponga el propio tratado internacional. Asimismo, cuando tengan por ámbito materias propias de su competencia podrán celebrarlos las Ciudades Autónomas y las Entidades Locales».

En ejercicio de sus competencias y en el marco de las leyes, los municipios pueden realizar actuaciones en el exterior, actuaciones que constituyen una parte de la acción exterior del Estado. Es cierto que estas actuaciones deben ejercerse en el marco de las directrices, fines y objetivos fijados por el Gobierno, pero estas directrices, fines y objetivos, no pueden desconocer que hoy el exterior ya no es aquel lugar lejano y ajeno que fue en algún momento. La globalización y las comunicaciones han hecho, que todo lo que ocurre en cualquier lugar, afecte a todos, incluidos los municipios y demás entes locales y que el exterior constituya hoy un ámbito en el que los entes locales, pueden y deben actuar en el marco de sus competencias, en la forma establecida en el ordenamiento jurídico, y también en la que, derive de los propios organismos internacionales. Los municipios y demás entes locales, también tienen obligaciones derivadas de las normas internacionales.

Es cierto, que la Constitución Española en el artículo 96.1 vincula la consideración de derecho interno, al cumplimiento de determinadas formalidades y a la publicación del contenido de los tratados en el Boletín Oficial del Estado, y también que la regla general es, que el sujeto que asume las obligaciones respecto al exterior es el 
Gobierno de España, al corresponderle conforme al artículo 149 de la Constitución, la competencia exclusiva en materia de relaciones internacionales puesto que «distribuye internamente las obligaciones en el marco de las competencias», aunque, como señala el preámbulo de la Ley 25/2014 de 27 de noviembre, de Tratados y otros Acuerdos internacionales:

«No obstante, las Comunidades Autónomas tienen competencia para desplegar ciertas actividades de acción exterior, entre las que cabe, por ejemplo, la celebración de acuerdos internacionales no normativos. También disponen de competencia para celebrar acuerdos internacionales administrativos, en concreción o ejecución de un tratado. Gozan además de competencias, en otros aspectos de la acción exterior, que también tienen consecuencias en la propia política exterior del Estado en materia de celebración de tratados internacionales...».

Conforme a los artículos 7, 44, 52 y 53 de la ley citada, los entes locales pueden igualmente, celebrar acuerdos internacionales administrativos y no normativos.

Todo ello determina que los entes locales, no solo pueden actuar en el exterior, sino que tendrán que hacerlo en muchas ocasiones, cada vez más, en el ejercicio de sus competencias aunque sometiéndose a las normas que obligan al Estado. Lo que implica que también habrán de seguir las obligaciones o recomendaciones, derivadas de las declaraciones internacionales, en los asuntos que les atañen.

La Agenda 2030 se refiere al logro de objetivos y metas, más allá de los territorios de cada Estado, se refiere a actuaciones en el interior y en el exterior. Las metas se han de lograr, tanto dentro de los países desarrollados como en los países en desarrollo. Hay una sola finalidad, pero dos marcos territoriales de actuación.

Internamente, la primera actuación es la de implementar, aquí, en nuestro país, los Objetivos de Desarrollo Sostenible (en adelante los ODS).

Los ODS habrá que integrarlos en las políticas públicas, a nivel Estatal, autonómico y local. Para ello, por lo que se refiere a la Administración Local, habrá que establecer planes de actuación en cada municipio y en cada entidad local, para alinear el ejercicio de sus competencias al logro de los ODS. Pero no sólo eso, en algunos casos será necesario además reorientar las políticas e incluso establecer nuevas acciones, porque para el logro de los ODS, no basta con alinear lo que ya se está haciendo, además habrá que examinar si alguna meta u objetivo, que actualmente no forma parte de sus acciones, es posible abordarla en el marco de las competencias de las que se es titular y si ello es así añadir estas acciones a los planes de actuación. La implementación de los ODS supone orientar todas las políticas públicas que se ejerzan a la consecución de los ODS, de todos a la vez. Pero hay más, la implementación de los ODS, no es sólo tarea del sector público, es también tarea de la sociedad civil y ahí es donde los entes locales por su proximidad, habrán de llevar a cabo acciones tanto para su divulgación como para conseguir incorporar a todos al proceso.

La segunda actuación necesaria es, dotar suficientemente los presupuestos, tanto para las nuevas actividades, como para seguir atendiendo la Cooperación Internacional para el Desarrollo en el exterior. El ODS 17 se refiere a «destinar el 0,7 \% del ingreso nacional bruto a la asistencia oficial para el desarrollo y del 0,15\% al 0,20 \% del ingreso nacional bruto, a la asistencia oficial para el desarrollo de los países menos adelantados; y alentar a los proveedores de asistencia oficial para el desarrollo a que consideren fijar una meta para destinar al menos el 0,20\% del ingreso nacional bruto a la asistencia oficial para el desarrollo de los países menos adelantados».

Se trata de dos acciones complementarias, la primera es total, abarca a todas las actuaciones en el territorio de cada administración pública; la segunda, más allá de los recursos, supone orientar igualmente la ayuda a un territorio o territorios concretos en el exterior destinandola a acciones que contribuyan al logro de los ODS. Una y otra son complementarias, porque lo que se aborda con la declaración, es una agenda mundial, que se ha de lograr actuando en el interior de cada país y en el exterior.

\section{LA AGENDA 2030 PARA EL DESARROLLO SOSTENIBLE}

La Asamblea General de Naciones Unidas, en Septiembre de 2015, aprobó con el título «Transformar nuestro mundo» la Agenda 2030 para el Desarrollo Sostenible, que contiene 17 objetivos y 169 metas que los firmantes se comprometen a alcanzar entre 2016 y 2030.

España ha suscrito esa agenda, que según se lee en su texto, «debe orientar sus actuaciones en el marco de los compromisos que contiene». Compromisos que, además de a todos los Estados, conciernen a todos los ciuda- 
danos, a todas las empresas, a todas las organizaciones a todas las administraciones públicas y por tanto a todos los municipios y demás entes locales de nuestro país.

La Agenda 2030 incluye, tácitamente a los municipios y demás entes locales, como actores para la consecución de los objetivos previstos. Comprende diversas actuaciones y metas, algunas de las cuales como se ha dicho, hasta hace muy poco, se consideraban excluidas del ámbito local. Obviamente el marco de actuación de los entes locales para el cumplimiento de los ODS son sus propias competencias.

Los Objetivos de Desarrollo Sostenible, se elaboraron y formularon después de que concluyera el periodo (2001/2015) para la consecución de los Objetivos de Desarrollo del Milenio (ODM). En cierto modo y en algunas de sus partes, los ODS, pueden ser considerados como una continuidad o una segunda parte de estos últimos, aunque van mucho más allá.

La formulación de los ODM en el año 2000, supuso el mayor compromiso global contra la pobreza, mediante el reconocimiento de la responsabilidad colectiva de respetar y defender los principios de la dignidad humana, la igualdad y la equidad, en el plano mundial. Su objetivo era crear un entorno propicio al desarrollo y la eliminación de la pobreza y aun cuando, se formularon y se previó la consecución de los objetivos, conjuntamente entre países desarrollados y en desarrollo, esencialmente se trataba de actuar en estos últimos. El escenario donde actuar se situaba principalmente en el Sur, porque los problemas que se trataba de resolver estaban en el Sur. El Norte rico y desarrollado actuaba en el Sur pobre y en desarrollo. Ahora la Agenda 2030, contempla también la especial situación de los países pobres, pero el ámbito de actuación aparece claramente delimitado a todos los países y a todos los actores.

Los ODS son globales. No se contempla solo un Norte rico, que resuelve problemas o que aporta recursos, para un Sur pobre. Se parte del reconocimiento de que los problemas son globales y afectan a toda la humanidad. Como dice el preámbulo de la Agenda 2030, «afectan a todas las personas y a todo el planeta». Los diecisiete ODS con las 169 metas que los integran, abordan la práctica totalidad de los problemas a que se enfrenta actualmente la humanidad.

Por su contenido y su formulación, por los objetivos que persigue y por los actores a los que compromete, la Agenda 2030 se parece mucho más a un programa de Gobierno de la humanidad que a una agenda. Es un programa de Gobierno de la Humanidad, para cuyo cumplimiento los gobiernos de los países firmantes deberán, cuanto menos, inspirarse, al elaborar e implementar sus políticas, sus programas de gobierno, sus acciones y además rendir cuenta de su cumplimiento.

«La Agenda 2030 se sitúa en el centro de la gobernabilidad mundial, ya no son acciones y objetivos que se formulan y ejecutan desde las agencias y unidades de Cooperación Internacional para el desarrollo de los Estados firmantes, sino que son políticas generales, a integrar en todas y cada una de las acciones de gobierno, en todos sus niveles. La Agenda 2030 difumina la línea divisoria entre políticas domésticas, política exterior y política de ayuda al desarrollo»³.

En contra de lo que ocurría con los ODM, ahora la responsabilidad del cumplimiento de los ODS, es responsabilidad de todas las unidades de cada Administración. Hasta el punto de que la dirección y coordinación del cumplimiento, debería residenciarse en la Presidencia del Gobierno o en el órgano al que se le atribuya la dirección y coordinación de la política en su integridad, sin perjuicio de que los aspectos que constituyen la Cooperación Internacional para el Desarrollo, en sentido estricto (en adelante CID), se encomienden a un departamento diferenciado. Gabriel Ferrero, propone, para articular la nueva política que suponen los ODS, «Crear un Ministerio de Desarrollo Sostenible, con rango de Vicepresidencia del Gobierno encargado de formular y desarrollar esta política» "Oxfam Intermón (OI) y Unicef, se refieren a elaborar una estrategia nacional y proponen crear una vicepresidencia para coordinar la implementación de los ODS»5.

Es cierto, que como ocurre siempre con las declaraciones de los organismos multilaterales, su carácter queda entre la obligación y la recomendación, extremo que en el caso de la Agenda 2030, no solo deriva de los artículos 10 y siguientes de la Carta de Naciones Unidas, sino de la propia declaración, que utiliza términos tales como procuraremos, reconocemos, puede hacerse realidad, apoyamos, etc. poco concluyentes y que dejan un amplio margen de actuación, aun cuando se prevén en la Agenda mecanismos de control, seguimiento y evaluación. Pese a ello, en esta ocasión, hay algunas señales que permiten ser optimistas, como por ejemplo la implicación de la UE y de

3 FERRERO, Gabriel (2016).

4 FERRERO, Gabriel (2016)

5 AGudo, Alejandra (2016).

REALA, Nueva Época, - N. 5, enero-junio 2016 - ISSN: 1989-8975 - DOI: 10.24965/reala.voi5.10347 
la Casa Blanca, en este último caso a través de las declaraciones del Presidente Obama en la Cumbre y el creciente número de países, que ya están adaptando sus políticas e instituciones a los ODS ${ }^{6}$.

La declaración contenida en la Agenda 2030, supone que la humanidad dispone para los próximos quince años de un plan de actuación, de unas directrices y de unos principios, para abordar la convivencia de las personas y las Naciones en un mundo cada vez más complejo y para enfrentarse y dar solución al hambre, a la educación, la pobreza, la desigualdad, el consumo, el cambio climático y así hasta los 17 ODS.

Es cierto, que la agenda no contiene compromisos concretos por parte de los firmantes, ni consecuencias para los incumplimientos. También es cierto que magnificas declaraciones y compromisos de la comunidad internacional, en esta y otras materias se vienen incumpliendo, pero haber sido capaces de redactar este documento, de suscribirlo y de que esté en vigor desde el 1 de enero de 2016, supone un enorme triunfo, un gran paso de la humanidad. Los municipios y demás entes locales están comprometidos, y deberán hacer grandes aportes para el logro de los objetivos, ya que son los titulares de competencias referidas a gran cantidad de las materias necesarias para el logro de los ODS. Para ello es fundamental que asuman el rol que el ordenamiento jurídico les atribuye al respecto, además de dotarse de nuevos instrumentos y capacidades.

\section{LOS OBJETIVOS QUE COMPRENDE LA AGENDA 2030}

Los ODS contenidos en la agenda aprobada son los siguientes:

1. Erradicar la pobreza en todas sus formas en todo el mundo.

2. Poner fin al hambre, conseguir la seguridad alimentaria y una mejor nutrición, y promover la agricultura sostenible.

3. Garantizar una vida saludable y promover el bienestar para todos y todas en todas las edades.

4. Garantizar una educación de calidad inclusiva y equitativa, y promover las oportunidades de aprendizaje permanente para todos.

5. Alcanzar la igualdad entre los géneros y empoderar a todas las mujeres y niñas.

6. Garantizar la disponibilidad y la gestión sostenible del agua y el saneamiento para todos.

7. Asegurar el acceso a energías asequibles, fiables, sostenibles y modernas para todos.

8. Fomentar el crecimiento económico sostenido, inclusivo y sostenible, el empleo pleno y productivo, y el trabajo decente para todos.

9. Desarrollar infraestructuras resilientes, promover la industrialización inclusiva y sostenible, y fomentar la innovación.

10. Reducir las desigualdades entre países y dentro de ellos.

11. Conseguir que las ciudades y los asentamientos humanos sean inclusivos, seguros, resilientes y sostenibles.

12. Garantizar las pautas de consumo y de producción sostenibles.

13. Tomar medidas urgentes para combatir el cambio climático y sus efectos.

14. Conservar y utilizar de forma sostenible los océanos, mares y recursos marinos para lograr el desarrollo sostenible.

15. Proteger, restaurar y promover la utilización sostenible de los ecosistemas terrestres, gestionar de manera sostenible los bosques, combatir la desertificación y detener y revertir la degradación de la tierra, y frenar la pérdida de diversidad biológica.

16. Promover sociedades pacíficas e inclusivas para el desarrollo sostenible, facilitar acceso a la justicia para todos y crear instituciones eficaces, responsables e inclusivas a todos los niveles.

17. Fortalecer los medios de ejecución y reavivar la alianza mundial para el desarrollo sostenible.

La Agenda 2030 que contiene los ODS, es universal, abarca a todo el planeta y a toda la población mundial. Así resulta del preámbulo del acuerdo que aprueba la Agenda.

6 FERRERO, Gabriel (2016).

REALA, Nueva Época, - N. ${ }^{\circ}$ 5, enero-junio 2016 - ISSN: 1989-8975 - DOI: 10.24965/reala.voi5.10347 
Sus objetivos son las personas, el planeta y la prosperidad, a la vez que fortalecer la paz universal. Se parte de reconocer en el preambulo, «que la erradicación de la pobreza es el mayor desafío a que se enfrenta el mundo y constituye un requisito indispensable para el desarrollo sostenible».

El plan que se contiene en la agenda, será implementado en todos los países y partes interesadas, mediante una alianza de colaboración.

La Agenda pretende hacer realidad los derechos humanos de todos, la igualdad de género, el empoderamiento de las mujeres y niñas... Los objetivos además, son de carácter integrado e indivisible y conjuntan las tres dimensiones del desarrollo sostenible: económica, social y ambiental.

Con mayor o menor intensidad, para el logro de los objetivos, hay tareas de la competencia de los municipios y demás entes locales que ya desarrollan y otras muchas que pueden incorporar, sin excesivo esfuerzo en sus políticas. Lo mismo cabe decir respecto a las 169 metas.

A lo largo de la historia los entes locales españoles, han sabido incorporar a sus políticas, lo necesario para atender los nuevos retos que han ido apareciendo, ahora, en el marco de sus competencias también deberán hacerlo implementando la Agenda 2030.

\section{NATURALEZA JURIDICA DE LA AGENDA}

Para delimitar el alcance de los ODS, procede indagar acerca de la naturaleza jurídica de la declaración contenida en la Agenda 2030.

Si los entes locales, también el resto de actores públicos, han de aplicar la Agenda, es necesario conocer que es en términos jurídicos la declaración, así como delimitar si contiene aspectos normativos y obligacionales o no, y para el supuesto de que la respuesta sea negativa, qué efectos tiene o como ha de aplicarse o incorporarse al ordenamiento y a las políticas públicas y finalmente, que se puede deducir de la lectura de la propia declaración, qué términos utiliza, para a través de ellos, llegar a la voluntad expresada en el documento y conocer cuál fue la voluntad de los firmantes.

Respecto a si es o no una norma jurídica, que obliga internamente, esta es una cuestión que en nuestro ordenamiento jurídico plantea pocas dudas. El artículo 96 de la Constitución establece que:

«los tratados internacionales válidamente celebrados, una vez publicados oficialmente en España, formarán parte del ordenamiento interno. Sus disposiciones sólo podrán ser derogadas, modificadas o suspendidas en la forma prevista en los propios tratados o de acuerdo con las normas generales del Derecho internacional».

Si esto es así para los Tratados, no cabe duda que la Agenda 2030, que se contiene en una declaración aprobada mediante una resolución, cuyo rango es muy inferior a un Tratado, no forma parte de nuestro derecho interno y por tanto no tendría carácter obligatorio.

La declaración ha sido dictada en el ámbito internacional después de un largo proceso, en el que han participado multitud de actores y ha sido suscrita por los 193 países de las Naciones Unidas, por lo que es difícil considerar que se trata de una mera manifestación de voluntad o de deseo. Por lo que es necesario plantearse, cuál pueda ser su ubicación dentro del ordenamiento jurídico, y como integrar en la acción pública y privada a que se refieren los objetivos, la manifestación de voluntad expresada por los Estados en la firma del documento.

El alcance de las declaraciones de los organismos internacionales, no ha sido excesivamente estudiado, si bien hay algunos autores que se han preocupado de ello.

«Hay que advertir que en la doctrina, existe imprecisión jurídica sobre este tema, (el valor jurídico de las resoluciones de las organizaciones internacionales), que la práctica internacional de las Naciones Unidas y de las organizaciones internacionales está precisando. No hay un régimen común de las organizaciones internacionales, dado que dependen de su tratado constitutivo y por esto es difícil intentar una respuesta común aplicable en todos los casos»?.

«No obstante el número creciente y la importancia cada vez mayor que tienen las resoluciones en la vida internacional, poco se ha avanzado, desde que se crearon los primeros organismos internacio-

7 MONROY CABRA, Marco Gerardo. (2007).

REALA, Nueva Época, - N. ${ }^{\circ}$ 5, enero-junio 2016 - ISSN: 1989-8975 - DOI: 10.24965/reala.voi5.10347 
nales, en la determinación de su naturaleza y en la precisión de su alcance y efectos... Hoy en día, no existe por decirlo así, una teoría general de las resoluciones internacionales. Aparte de describirlas, como expresión formal del parecer de un organismo internacional, sería difícil señalar ciertos rasgos característicos, que permitieran integrar una definición significativa y suficientemente precisa... los Organismos Internacionales pueden tomar decisiones a través de sus diversos órganos. Se usan diversos términos para designar a dichos actos o decisiones: resolución, recomendación, decisión, acuerdo, regulación, ordenanza, opinión, etcétera. La evidente proliferación de los Organismos Internacionales, en épocas recientes, y su constante actividad dentro de la comunidad internacional, confieren una creciente importancia a sus decisiones. Por consiguiente, es interesante evaluar los efectos jurídicos de éstas... en su mayoría, las resoluciones de los organismos internacionales, no crean obligaciones para los Estados. Salvo lo referido a su contribución para la formación de las normas consuetudinarias... Los organismos internacionales pueden adoptar decisiones internas y externas, en estas últimas: ha de observarse sin embargo, que son excepción las Organizaciones Internacionales, capacitadas para adoptar actos jurídicos de contenido normativo. En general, sus resoluciones de carácter externo o funcional son recomendaciones, mediante las que invita a los Estados miembros para comportarse en un determinado sentido, incumbiéndole a éstos únicamente la obligación de apreciar de buena fe la posibilidad de observar lo recomendado» ${ }^{8}$.

La afirmación, según la cual, la Agenda 2030 y por tanto los objetivos, las metas y en definitiva el Plan de Acción que contiene, son una mera recomendación, a mi juicio no puede aceptarse. Aunque formalmente pueda parecerlo, no lo es, porque la declaración, contiene inequívocamente, la voluntad de los Estados firmantes de resolver cuestiones esenciales para la humanidad y porque más allá de referencias abstractas, contiene acciones bien definidas, tanto en cantidad como en el tiempo en que han de lograrse.

El hecho de que las voluntades coincidentes de los Estados firmantes, no tengan la forma de Tratado u otra figura semejante, suscrita con todas las formalidades, que la haga «jurídicamente correcta» no la convierte en inoperante.

Ante los retos a que se enfrenta la humanidad y que la Agenda se propone resolver, los Estados a través de Naciones Unidades, han reaccionado y han abierto vías de actuación y establecido reglas y principios a cumplir y lo han hecho así, mediante una declaración, porque al fin y al cabo una de las características del derecho internacional, es que uno de los aspectos esenciales de las normas jurídicas, la coactividad, queda profundamente diluida también en los Tratados u otros instrumentos, por lo que materialmente ante el incumplimiento de un tratado, la reacción puede no ser tan diferente al incumplimiento de una declaración.

La evolución de las relaciones internacionales, han hecho proliferar la figura de las declaraciones, que como la Agenda 2030, utilizan vías diferentes a las tradicionales para llegar a puntos de encuentro de un modo más ágil, amplio, abierto y comprometido que lo hubiera podido hacer un Tratado. Las recomendaciones contenidas en la Agenda 2030, sin duda exigían no solo llegar a puntos de encuentro, sino hacerlo sin demora, porque no olvidemos que los objetivos y las metas que se contienen, se refieren a las personas, el planeta, la prosperidad y la paz y tratan de hacerlo casi a contra reloj, porque es urgente actuar en esos ámbitos y hacerlo ya. Al decir de los científicos en algunas cuestiones, como en el clima, de modo muy urgente. La declaración, más allá de su formalidad jurídica, sin duda es necesaria y su contenido va a incorporarse a las políticas públicas en muchos países para el logro de los objetivos.

Los firmantes de la Agenda, pretenden materialmente un efecto que va más allá de la recomendación.

«... Es preciso poner de presente, que el valor y los efectos jurídicos de una resolución, no son solamente aquellos que se derivan formalmente de este instrumento como tal (la resolución), sino que deben ser buscados igualmente, en aquellos que derivan de su contenido, es decir de la operación jurídica que tiene el instrumento. Es la distinción del derecho romano entre instrumentum y el negotium» 9 .

Aunque en la Agenda 2030 el instrumentum pueda no ser formalmente el más adecuado para obligar, el negotium está muy claro, la agenda contiene el acuerdo de voluntades soberanas, «en favor de las personas, el planeta y la prosperidad... » (preámbulo de la declaración).

8 Carrillo Salazar, Augusto (2014).

9 ABI-SAAB, Georges (1971).

REALA, Nueva Época, - N. ${ }^{\circ}$ 5, enero-junio 2016 - ISSN: 1989-8975 - DOI: 10.24965/reala.voi5.10347 
«En cuanto a las recomendaciones, la regla general es la no obligatoriedad jurídica. Sin embargo, éstas tienen impacto político y a veces, imponen determinadas obligaciones, como notificar el curso que se les ha dado, dar informes periódicos o respuesta a cuestionarios, explicar las razones por las que no se han cumplido, etc. Sin embargo, como dice Díez de Velasco, estas recomendaciones se diferencian de las decisiones obligatorias, en que los Estados miembros no están obligados a ejecutar su contenido, puesto que las obligaciones que establecen son de orden procedimental más que relativas al fondo del asunto» ${ }^{10}$.

El impacto político es uno de los elementos que confiere a las declaraciones, obligatoriedad en cuanto al negotium. Más allá de la obligatoriedad jurídica, del cumplimiento de los compromisos internacionales, contenidos en acuerdos o declaraciones, como la de la Agenda, depende el prestigio de los Estados y el rol que desempeñan en el concierto internacional.

«La obligatoriedad jurídica de las resoluciones de las organizaciones internacionales, ha sido estudiada en parte de la doctrina bajo la noción de soft law o derecho programático, formado por normas cuyo contenido resulta impreciso y vago e insuficiente para llegar a ser regla del derecho»"

En Derecho Internacional, la expresión soft law no posee una definición unívoca, pero apunta a aquellos instrumentos cuya juridicidad o fuerza vinculante es ambigua o se cuestiona. Normalmente se aplica a textos en los que suelen concurrir, a la vez, el carácter no jurídicamente vinculante y una cierta relevancia jurídica ${ }^{12}$.

«La expresión soft law... de una parte, se aplica a instrumentos... en los que suelen concurrir dos elementos, el carácter no jurídicamente vinculante, pero una cierta relevancia jurídica. Por otra, se usa para describir el contenido de algunos acuerdos, jurídicamente vinculantes que por la vaguedad en su formulación o por la discrecionalidad que confieren a las partes en su cumplimiento, en realidad no imponen obligaciones exigibles (soft law material). Por tanto, dentro de la propia expresión se suele distinguir entre reglas jurídicas (legal soft law), que decantan la balanza hacia el law, y no jurídicas (non-legal soft law), que lo harían hacia el soft.

Así, el soft law es una zona intermedia digna de consideración, por sus efectos, situada entre la norma vinculante y la completa ausencia de significación jurídica... En nuestra opinión, es justamente entre esas dos posiciones donde se ubica el soft law, ya que es cierto que las partes han cooperado, más allá de la mera negociación política, para llegar a un pacto por el que no se quieren obligar de la misma forma que con un tratado internacional tradicional»»3.

La declaración de la Agenda 2030 es un pacto muy detallado, sobre materias de gran calado para la humanidad. Pacto que se contiene en un instrumento que formalmente no está dotado de la fuerza de un Tratado, pero es mucho más que una declaración, por lo que su aplicabilidad debe derivar también de los términos de la propia declaración.

Si examinamos la propia declaración, en su parte referida a la Nueva Agenda señala en los puntos:

1. «Cada país enfrenta desafíos específicos».

2. «Es necesario empoderar a las personas vulnerables».

3. «Nos comprometemos a poner fin a la pobreza».

4. «Nos comprometemos a proporcionar una educación de calidad».

5. «Para promover la salud... debemos lograr la cobertura sanitaria universal».

6. «Procuraremos sentar unas bases económicas sólidas en todos nuestros países».

7. «Nos comprometemos a efectuar cambios fundamentales en la manera en que nuestras sociedades consumen y producen bienes y servicios».

10 MONROY CABRA, Marco Gerardo (2007).

11 DupUY, René Jean (1999).

12 CHICHARRO, Alicia (2012)

13 ChICHARRo, Alicia (2012). 
8. «Reconocemos la positiva contribución de los migrantes al crecimiento inclusivo».

9. «Se insta encarecidamente a los Estados que se abstengan de promulgar y aplicar medidas incompatibles con el derecho internacional».

En la parte referida Objetivos de Desarrollo sostenible y metas señala en los puntos:

«55. Los ODS y sus metas son de carácter integrado e indivisible, de alcance mundial y de aplicación universal, tienen en cuenta las diferentes realidades, capacidades y niveles de desarrollo de cada país y respetan sus políticas y prioridades nacionales. Si bien las metas expresan las aspiraciones a nivel mundial, cada gobierno fijará sus propias metas nacionales...».

Este apartado confiere una amplia discrecionalidad a los firmantes, en el logro de los ODS, lo cual por otra parte es lógico. Cada país, y dentro de este en cada territorio parte de carencias, recursos y realidades muy diferentes. Establecer un sistema homogéneo no es posible. Sin embargo esto puede debilitar su fuerza vinculante, ya que una regla diferente para adaptar los compromisos a cada realidad puede desvirtuar la aplicación. Se pone de manifiesto lo que ya hemos señalado que en el ámbito de sus respectivas competencias, las Administraciones Públicas deberían establecer sus propios criterios para el desarrollo de los desafíos que se contienen, criterios y reglas que deberían ser el resultado de un amplio consenso entre todos los actores. La Federación Española de Municipios y Provincias junto a otros actores locales, podría ser un ámbito muy oportuno para abrir una reflexión.

«56. Al acordar estos Objetivos y metas reconocemos que cada país enfrenta desafíos específicos para lograr el desarrollo sostenible, y recalcamos los problemas especiales con que tropiezan los países más vulnerables..., así como los países de ingresos medianos... ».

«57. Reconocemos que siguen sin existir datos de referencia para varias metas y pedimos mayor apoyo para fortalecer la recopilación de datos y la creación de capacidad en los Estados Miembros, a fin de establecer bases de referencia nacionales y mundiales cuando aún no existían... ».

Nuevamente partiendo de la realidad de los hechos, se reconocen las dificultades de algunos países lo que supone flexibilizar sus compromisos, a la vez que se reconoce, la necesidad de fortalecer la formación de las personas y construir instrumentos para la aplicación de la agenda singularmente para la recopilación de datos que permitan conocer los avances.

«59. Reconocemos que cada país dispone de diferentes enfoques, visiones de futuro, modelos e instrumentos, en función de sus circunstancias y prioridades nacionales, para lograr el desarrollo sostenible y reafirmamos que el planeta Tierra y sus ecosistemas son nuestro hogar común y que "la Madre Tierra" es una expresión corriente en muchos países y regiones».

Se remarca aquí el carácter diverso y antiformalista de las vías y procedimientos, para el cumplimiento de los ODS. Cada país ha de enfrentar su logro, desde su realidad. Pero si lo ha de hacer cada país, ello implica que habrá de establecer un mínimo de reglas comunes que permitan conocer los avances. A ello conduce el establecer indicadores comunes acerca del logro de los objetivos, indicadores respecto a los que se han de ordenar las acciones. Estos indicadores deben orientarse respecto a los que ha de aprobar NU.

\section{BIBLIOGRAFÍA}

ABI-SAAB, Georges, (1971), “Les sources du Droit Internacional. Essai de déconstruction”, en El derecho internacional en un mundo en transformación: Liber Amicorum en honor de Eduardo Jiménez de Aréchaga, tomo 1. Les résolutions dans la formation du droit international de développement, Geneve, IUHEI.

ABOGADA DEL ESTADO (2014), Informe de 10 de diciembre de la Abogada del Estado http://www.femp.es/ files/3580-995-fichero/Dictamen\%20de\%20la\%20Abogac\%C3\%ADa\%20General\%20del\%20Estado.pdf.

AGudo, Alejandra (2016), “La Agenda de desarrollo sobrevuela la política española y pide aterrizar”, El Pais, Planeta futuro, febrero.

ASAMBLEA GENERAL NACIONES UNIDAS (2015), Transformar nuestro mundo: La Agenda 2030 para el Desarrollo Sostenible. http://www.cooperacionespanola.es/sites/default/files/agenda_2030_desarrollo_sostenible_ cooperacion_espanola_12_ago_2015_es.pdf.

CARRILlo SALAzAR (2014) Augusto, Resoluciones de los organismos internacionales. Página Web a) Bibliografía: https://independent.academia.edu/AugustoCarrilloSalazar. 
Chicharro, Alicia (2012), Universidad Pública de Navarra. "El carácter de soft law de los instrumentos internacionales sobre desarrollo sostenible". Congreso Internacional de Estudios del Desarrollo Desafíos de los Estudios del Desarrollo. http://congresoreedes.unican.es/actas/PDFs/69.pdf.

DUPUY, Rene Jean (1999), “Droit declaratoire et Droit Programmatoire. De a coutume sauvage a la 'Softlaw”, en Dialectiques du Droit Internacional, s.I., In stitut de la paix et du Developpment, A Pedome.

Ferrero, Gabriel (Pnud) (2016), Diario El País, Planeta Futuro, 24 febrero.

GARCIA MATIES, Rafael (2015), "La cooperación internacional para el desarrollo por los entes locales, tras la reforma operada por la Ley 27/2013". Revista de estudios de la Administración Local y Autonómica (REALA) n. ${ }^{\circ}$ 4/2015.

MonRoy CABRA, Marco Gerardo (2007), Ensayos de teoría constitucional y derecho internacional. Valor Jurídico de las resoluciones de las organizaciones internacionales, Editorial Universidad del Rosario, edición 1, pág. 139. 